\title{
Morphological Spectrum and Pathological Parameters of Type 2 Endometrial Carcinoma: A Comparison With Type 1 Endometrial Cancers
}

\author{
Atif A. Hashmi ${ }^{1}$, Syeda N. Iftikhar ${ }^{1}$, Javaria Ali ${ }^{1}$, Fatima Shaheen ${ }^{2}$, Farhath Afroze ${ }^{3}$, Abira Imran ${ }^{4}$ \\ 1. Pathology, Liaquat National Hospital and Medical College, Karachi, PAK 2. Internal Medicine, MNR Medical College, \\ Hyderabad, IND 3. Internal Medicine, Deccan College of Medical Sciences, Hyderabad, IND 4. Statistics, Liaquat \\ National Hospital and Medical College, Karachi, PAK
}

Corresponding author: Atif A. Hashmi, atifhashmi345@gmail.com

\section{Abstract}

\section{Introduction}

Endometrial cancers (ECs) are the most common gynecological malignancies. Based on morphology and pathogenesis, ECs are segregated into type 1 and 2 ECs. Types 1 ECs are those tumors that are estrogendriven, whereas type 2 ECs are more aggressive and are independent of hormonal status. In the proposed study, we evaluated the clinicopathological parameters of type 2 ECs and its comparison with type 1 ECs.

\section{Methods}

We retrospectively analyzed seven-year data from archives of pathology, Liaquat National Hospital, from January 2013 to December 2019. All patients underwent radical surgeries for diagnosed EC on endometrial biopsy. All specimens were of total abdominal hysterectomy with bilateral salpingo-oophorectomy, omentectomy, and peritoneal sampling, along with pelvic lymphadenectomy. Records regarding tumor type, grade, depth of myometrial invasion, and ovarian, omental, nodal, and parametrial involvement were assessed.

\section{Results}

A total of 129 cases of ECs were included in the study. The mean age of the patients was $57.6 \pm 9.3$ years. Majority of the cases were type 1 ECs (82.2\%). The most common histological type of EC was endometrioid (82.2\%) followed by serous carcinoma (10.1\%). Most of the tumors were grade 1 (42.6\%) and the International Federation of Gynecology and Obstetrics (FIGO) stage I (72.8\%). Nodal metastases were present in eight cases (6.2\%) and adnexal involvement was present in 12 cases (9.3\%). We found a significant association of the type of EC with lymphovascular invasion, nodal metastasis, and adnexal involvement, whereas no significant association of EC type was seen with other clinicopathological characteristics.

Review began 10/08/2020 Review ended 10/13/2020 Published 10/18/2020

๑) Copyright 2020

Hashmi et al. This is an open access article distributed under the terms of the Creative Commons Attribution License CC-BY 4.0., which permits unrestricted use, distribution, and reproduction in any medium, provided the original author and source are credited.

\section{Conclusions}

Type 1 EC was the most frequent subtype of EC in our study. On the other hand, type 2 EC was significantly associated with nodal metastasis, lymphovascular invasion, and adnexal involvement, signifying the poor prognostic significance of this group of EC.

Categories: Obstetrics/Gynecology, Pathology, General Surgery

Keywords: endometrial carcinoma, endometrioid carcinoma, serous carcinoma, clear cell carcinoma, carcinosarcoma, type 1 endometrial carcinoma, type 2 endometrial carcinoma

\section{Introduction}

Endometrial cancers (ECs) are the most common gynecological malignancies. Based on morphology and pathogenesis, ECs are segregated into type 1 and 2 ECs. Types 1 ECs are those tumors that are estrogendriven, while type 2 ECs are more aggressive and are independent of hormonal status [1,2]. Type 1 ECs include endometrioid and mucinous carcinoma, whereas type 2 ECs include serous, clear cell, and carcinosarcoma histologies. Phenotypically, type 2 cancers appear more atypical under a microscope, and, molecularly, they are distinguished based on early p53 mutations. Apart from histological types, other prognostic parameters of EC include depth of myometrial invasion, parametrial involvement, lymphovascular invasion, and ovarian and nodal metastasis. In addition, the role of various diagnostic and prognostic biomarkers has been studied in ECs [3,4]; however, a large review concluded that currently there is no routinely used biomarker for diagnostic or prognostic purposes in ECs [5].

The segregation of epithelial endometrial tumors into two groups (type 1 and 2) could help clinicians and oncologists determine the response of different treatment modalities in these two separate prognostic 
groups of EC. Recently, a molecular-based classification of ECs has been proposed; however, underdeveloped and resource-limited countries with lack of molecular diagnostic facilities continue to rely on conventional histological parameters. In the proposed study, we evaluated the clinicopathological parameters of type $2 \mathrm{EC}$ and its comparison with type $1 \mathrm{EC}$.

\section{Materials And Methods}

We retrospectively analyzed seven-year data from archives of pathology, Liaquat National Hospital, from January 2013 to December 2019. All patients underwent radical surgeries for diagnosed EC on endometrial biopsy. All specimens were of total abdominal hysterectomy with bilateral salpingo-oophorectomy, omentectomy, and peritoneal sampling, along with pelvic lymphadenectomy. Records regarding tumor type, grade, depth of myometrial invasion, and ovarian, omental, nodal, and parametrial involvement were assessed.

Cases with unequivocal diagnosis of primary endometrial carcinoma were included in the study. Cases with post-neoadjuvant chemoradiation or secondary malignancies were excluded from the study. In addition, cases with non-epithelial (stromal) malignancies were also excluded from the study.

Gross examination of the specimens was performed according to standard protocols. Tumor dimension and extent of myometrial involvement were noted, and representative sections were submitted from the tumor. In cases where the tumor measured less than $2 \mathrm{~cm}$, whole tumor was submitted. In cases of larger than $2-\mathrm{cm}$ tumors, one section per centimeter of the tumor was submitted. In addition, representative sections from parametria, utero-cervical junction, cervix, fallopian tubes, ovaries, and the omentum were submitted. Pelvic lymph nodes were submitted entirely, and representative sections from the omentum were taken and submitted.

Immunohistochemical studies were conducted in selected cases of EC. Cytokeratin (CKAE1/3) immunostain was applied on all high-grade (grade 3) cases of EC to confirm epithelial nature of malignancy. In addition, p53 and napsin immunostains were performed for serous and clear cell carcinoma, respectively. In cases of suspicion of secondary malignancy, a panel of immunostains was applied, including CK7, CK20, TTF1, CDX2, GATA3, PAX8, and mamoglobin, to exclude metastatic carcinoma.

Data analysis was performed using Statistical Package for Social Sciences (SPSS) Version 26 (IBM Corp., Armonk, NY, USA). Chi-square test was used to check the association. P-values of $\leqslant 0.05$ were considered as significant.

\section{Results}

\section{Descriptive characteristics of the population under study}

A total of 129 cases of EC were included in the study. The mean age of the patients was $57.6 \pm 9.3$ years. Most of the patients were over 50 years of age (77.5\%) and postmenopausal (85.3\%). Majority of the cases were of type $1 \mathrm{EC}(82.2 \%)$. The most common histological type of EC was endometrioid (82.2\%) followed by serous carcinoma (10.1\%), carcinosarcoma (7\%), and clear cell carcinoma (0.8\%), as shown in Figures 1-4.






\section{Cureus}

FIGURE 1: Type 1 endometrial carcinoma: endometrioid carcinoma of the endometrium. (A) H\&E-stained sections at $100 \mathrm{X}$ magnification showing fused glandular configuration of tumor with focal solid area of growth (arrow). (B) 200X magnification showing gland formation (arrow). (C) 400X magnification showing low nuclear grade and absence of prominent nucleoli.

$\mathrm{H} \& \mathrm{E}$, hematoxylin and eosin

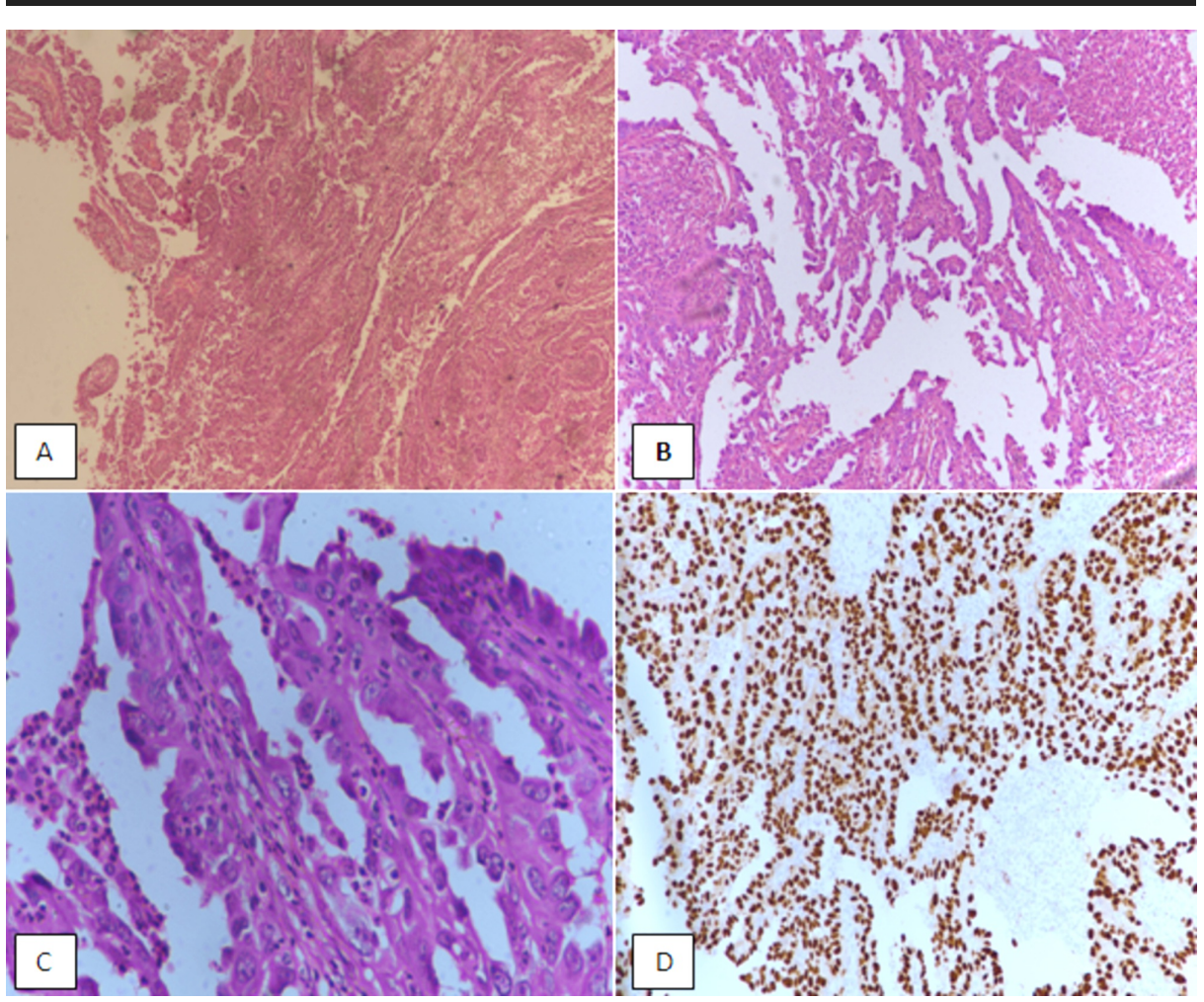

FIGURE 2: Type 2 endometrial carcinoma: serous carcinoma of the endometrium. (A) H\&E sections at $40 \mathrm{X}$ magnification showing diffuse growth of tumor with vague papillary configuration. (B) $100 \mathrm{X}$ magnification showing papillary architecture. (C) 400X magnification showing atypical nuclei and prominent nucleoli. (D) p53 immunostain showing diffuse nuclear positivity in tumor cells.

H\&E, hematoxylin and eosin 


\section{Cureus}

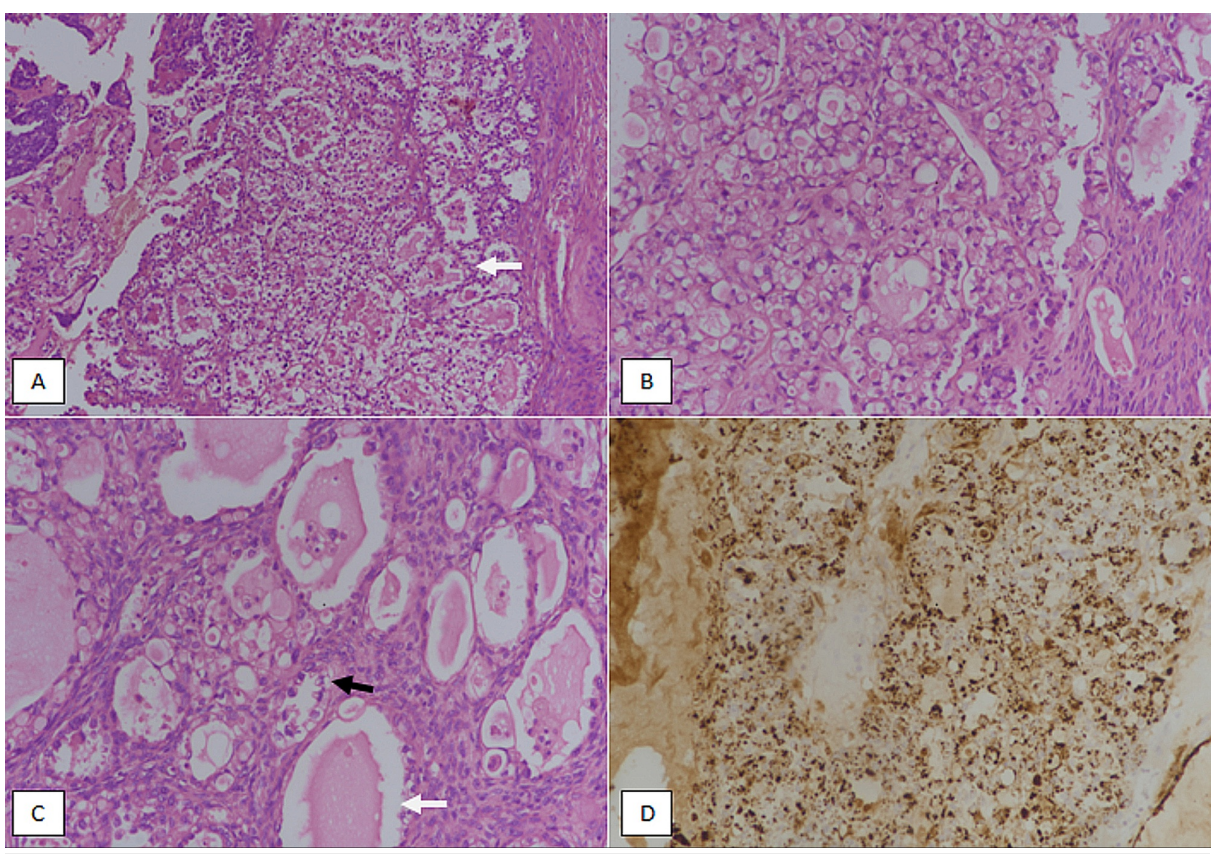

FIGURE 3: Type 2 endometrial carcinoma: clear cell carcinoma of the endometrium. (A) H\&E sections at 100X magnification showing tumor glands lined by clear cells (white arrow). (B) 200X magnification depicting tumor with tubuloglandular architecture lined by clear cells with intraluminal secretions. (C) 400X magnification showing hobnailing of cells (black arrow) and intra-luminal secretions (white arrow). (D) Napsin A immunostain showing diffuse positivity in tumor cells.

H\&E, hematoxylin and eosin

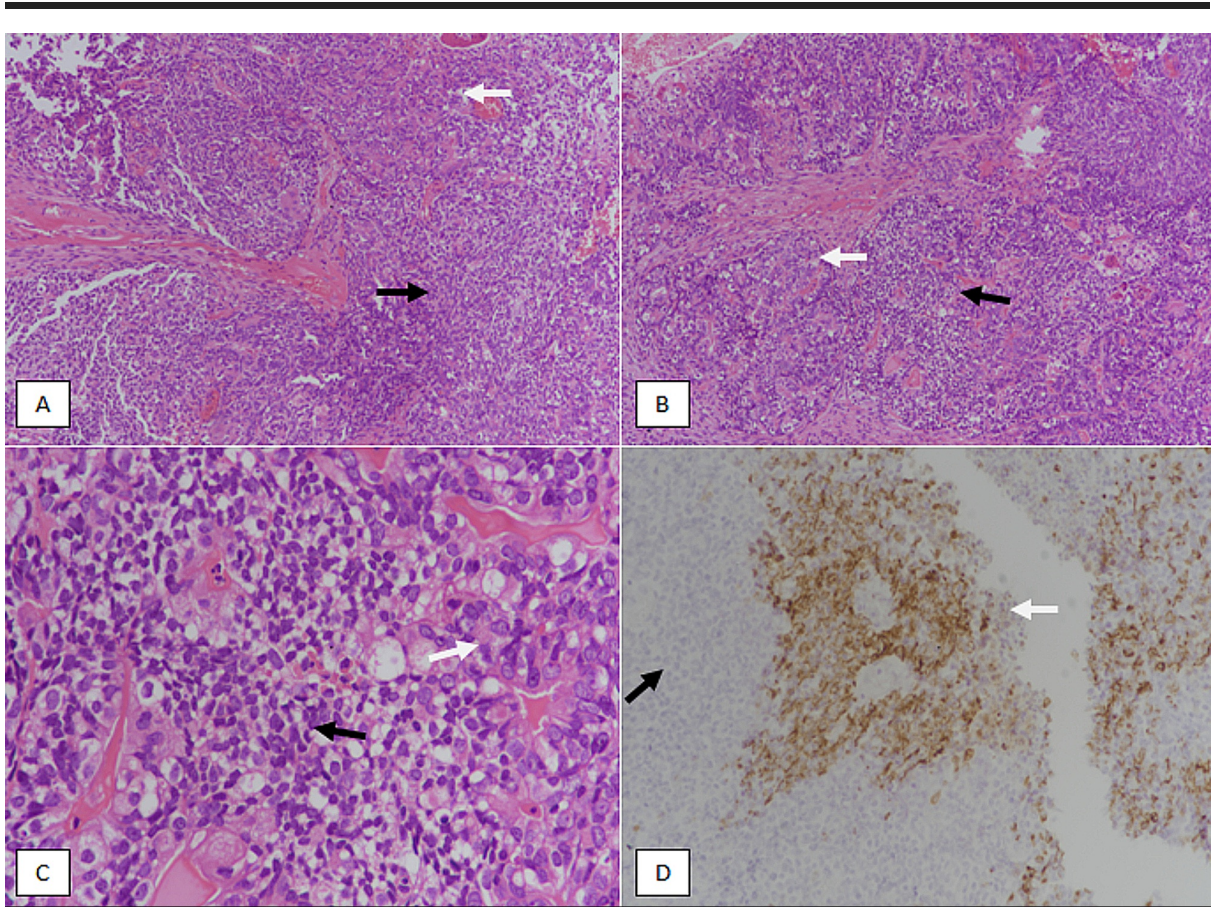

FIGURE 4: Type 2 endometrial carcinoma: carcinosarcoma of the endometrium. (A \& B) H\&E-stained sections at 100X magnification showing biphasic tumor with carcinomatous (white arrow) and sarcomatous components (black arrow). (C) 400X magnification 


\section{Cureus}

showing carcinomatous component (white arrow) with highly atypical nuclei and sarcomatous component (black arrow). (D) Cytokeratin (CKAE1/3) immunostain showing positive staining in carcinomatous component (white arrow) and negative staining in the sarcomatous component (black arrow).

H\&E, hematoxylin and eosin

Most of the tumors were grade 1 (42.6\%) and the International Federation of Gynecology and Obstetrics (FIGO) stage I (72.8\%). Nodal metastases were present in 8 (6.2\%) cases and adnexal involvement was present in 12 (9.3\%) cases, as shown in Table 1.

Clinicopathological characteristics

Age group

$\leq 50$ years

$>50$ years

Menopausal status

Premenopausal

Postmenopausa

Histological type

Endometrioid

Serous

Carcinosarcoma

Clear cell

Grade

2

FIGO stage

IA

IB

IIIA

IIIB

IV

T stage

T1

T2

T3

T4

Lymphovascular invasion

Present
Frequency (\%)

$29(22.5)$

$100(77.5)$

19 (14.7)

$110(85.3)$

$106(82.2)$

$13(10.1)$

$9(7.0)$

$1(0.8)$

55 (42.6)

42 (32.6)

$32(24.8)$

51 (39.5)

43 (33.3)

20 (15.5)

8 (6.2)

3 (2.3)

4 (3.1)

92 (71.3)

21 (16.3)

$12(9.3)$

$4(3.1)$

$10(7.8)$ 


\section{Cureus}

Absent

N stage

No

121 (93.8)

N1

N2

Myometrial invasion

Limited to the endometrium

More than half of the myometrium

Less than half of the myometrium

$52(40.3)$

Cervical invasion

\section{Present}

Absent

98 (76)

Adnexal involvement

Present

Absent

Tumor type

Type 1

106 (82.2)

Type 2

$23(17.8)$

TABLE 1: Descriptive statistics of population under study

FIGO, International Federation of Gynecology and Obstetrics; T, tumor; N, nodal

\section{Association of the type of endometrial carcinoma with clinicopathological characteristics}

We found a significant association of the type of EC with lymphovascular invasion, nodal metastasis, and adnexal involvement, whereas no significant association of EC type was seen with other clinicopathological characteristics. Although $21.7 \%$ of type 2 ECs were noted to have T stage T3/T4 compared to $10.4 \%$ of type 1 ECs, the difference was not statistically significant (Table 2).

\begin{tabular}{|c|c|c|c|}
\hline \multirow{2}{*}{ Clinicopathological characteristics } & \multicolumn{2}{|c|}{ Type of endometrial carcinoma } & \multirow{2}{*}{ p-Value } \\
\hline & Type 1 & Type 2 & \\
\hline \multicolumn{4}{|l|}{ Age group } \\
\hline$\leq 50$ years & $26(24.5)$ & $3(13)$ & \multirow{2}{*}{0.283} \\
\hline$>50$ years & $80(75.5)$ & $20(87)$ & \\
\hline \multicolumn{4}{|l|}{ Menopausal status } \\
\hline Premenopausal & 17 (16) & $2(8.7)$ & \multirow{2}{*}{0.523} \\
\hline Postmenopausal & 89 (84) & 21 (91.3) & \\
\hline \multicolumn{4}{|l|}{ FIGO stage } \\
\hline IA & 45 (42.5) & $6(26.1)$ & \multirow[b]{4}{*}{0.290} \\
\hline IB & 34 (32.1) & $9(39.1)$ & \\
\hline II & $16(15.1)$ & 4 (17.4) & \\
\hline & & & \\
\hline
\end{tabular}




\section{Cureus}

\begin{tabular}{|c|c|c|c|}
\hline IIIA & $7(6.6)$ & $1(4.3)$ & \\
\hline IIIB & $2(1.9)$ & $1(4.3)$ & \\
\hline IV & $2(1.9)$ & $2(8.7)$ & \\
\hline \multicolumn{4}{|l|}{ T stage } \\
\hline T1 & 78 (73.6) & $14(60.9)$ & \multirow{4}{*}{0.223} \\
\hline T2 & $17(16)$ & $4(17.4)$ & \\
\hline T3 & $9(8.5)$ & $3(13)$ & \\
\hline T4 & $2(1.9)$ & $2(8.7)$ & \\
\hline \multicolumn{4}{|l|}{ Lymphovascular invasion } \\
\hline Present & $4(3.8)$ & $6(26.1)$ & \multirow{2}{*}{$0.002^{*}$} \\
\hline Absent & $102(96.2)$ & $17(73.9)$ & \\
\hline \multicolumn{4}{|l|}{$\mathrm{N}$ stage } \\
\hline NO & $104(98.1)$ & $17(73.9)$ & \multirow{3}{*}{$<0.001^{*}$} \\
\hline N1 & $1(0.9)$ & $6(26.1)$ & \\
\hline N2 & $1(0.9)$ & $0(0)$ & \\
\hline \multicolumn{4}{|l|}{ Myometrial invasion } \\
\hline Limited to the endometrium & $6(5.7)$ & $0(0)$ & \multirow{3}{*}{0.731} \\
\hline More than half of the myometrium & $58(54.7)$ & $13(56.5)$ & \\
\hline Less than half of the myometrium & $42(39.6)$ & $10(43.5)$ & \\
\hline \multicolumn{4}{|l|}{ Cervical invasion } \\
\hline Present & $26(24.5)$ & $5(21.7)$ & \multirow{2}{*}{0.777} \\
\hline Absent & $80(75.5)$ & $18(78.3)$ & \\
\hline \multicolumn{4}{|l|}{ Adnexal involvement } \\
\hline Present & $7(6.6)$ & $5(21.7)$ & \multirow{2}{*}{$0.023^{\star}$} \\
\hline Absent & $99(93.4)$ & $18(78.3)$ & \\
\hline
\end{tabular}

\section{TABLE 2: Association of clinicopathological parameters with type of endometrial carcinoma}

Chi-square test was applied.

${ }^{*} \mathrm{p}$-Value significant as $\leq 0.05$.

FIGO, International Federation of Gynecology and Obstetrics; T, tumor; N, nodal

Similarly, serous carcinoma and carcinosarcoma were noted to have a significantly higher association with nodal metastasis and lymphovascular invasion compared to endometrioid carcinoma (Table 3). 


\section{Cureus}

\begin{tabular}{|c|c|c|c|c|c|}
\hline Premenopausal & $17(16)$ & $2(15.4)$ & $0(0)$ & $0(0)$ & \multirow{2}{*}{0.608} \\
\hline Postmenopausal & $89(84)$ & $11(84.6)$ & $9(100)$ & $1(100)$ & \\
\hline \multicolumn{6}{|l|}{ FIGO stage } \\
\hline IA & $45(42.5)$ & $1(7.7)$ & $4(44.4)$ & $1(100)$ & \multirow{6}{*}{0.198} \\
\hline IB & $34(32.1)$ & $6(46.2)$ & $3(33.3)$ & $0(0)$ & \\
\hline II & $16(15.1)$ & $3(23.1)$ & 1 (11.1) & $0(0)$ & \\
\hline IIIA & $7(6.6)$ & $1(7.7)$ & $0(0)$ & $0(0)$ & \\
\hline IIIB & $2(1.9)$ & $1(7.7)$ & $0(0)$ & $0(0)$ & \\
\hline IV & $2(1.9)$ & $1(7.7)$ & 1 (11.1) & $0(0)$ & \\
\hline \multicolumn{5}{|l|}{ T stage } & \multirow{5}{*}{0.383} \\
\hline T1 & 78 (73.6) & $7(53.8)$ & $6(66.7)$ & $1(100)$ & \\
\hline T2 & $17(16)$ & $3(23.1)$ & 1 (11.1) & $0(0)$ & \\
\hline T3 & $9(8.5)$ & $2(15.4)$ & $1(11.1)$ & $0(0)$ & \\
\hline T4 & $2(1.9)$ & $1(7.7)$ & 1 (11.1) & $0(0)$ & \\
\hline \multicolumn{6}{|l|}{ Lymphovascular invasion } \\
\hline Present & $4(3.8)$ & $4(30.8)$ & 2 (22.2) & $0(0)$ & \multirow{2}{*}{$0.003^{\star}$} \\
\hline Absent & $102(96.2)$ & $9(69.2)$ & $7(77.8)$ & $1(100)$ & \\
\hline \multicolumn{6}{|l|}{$\mathrm{N}$ stage } \\
\hline No & $104(98.1)$ & $8(61.5)$ & $8(88.9)$ & $1(100)$ & \multirow{3}{*}{$<0.001^{*}$} \\
\hline N1 & $1(0.9)$ & $5(38.5)$ & 1 (11.1) & $0(0)$ & \\
\hline N2 & $1(0.9)$ & $0(0)$ & $0(0)$ & $0(0)$ & \\
\hline \multicolumn{6}{|l|}{ Myometrial invasion } \\
\hline Limited to the endometrium & $6(5.7)$ & $0(0)$ & $0(0)$ & $0(0)$ & \multirow{3}{*}{0.708} \\
\hline More than half of the myometrium & $58(54.7)$ & 9 (69.2) & $4(44.4)$ & $0(0)$ & \\
\hline Less than half of the myometrium & $42(39.6)$ & $4(30.8)$ & $5(55.6)$ & $1(100)$ & \\
\hline \multicolumn{6}{|l|}{ Cervical invasion } \\
\hline Present & $26(24.5)$ & $4(30.8)$ & $1(11.1)$ & $0(0)$ & \multirow{2}{*}{0.698} \\
\hline Absent & $80(75.5)$ & 9 (69.2) & $8(88.8)$ & $1(100)$ & \\
\hline \multicolumn{6}{|l|}{ Adnexal involvement } \\
\hline Present & $7(6.6)$ & $3(2.31)$ & $2(22.2)$ & $0(0)$ & \multirow{2}{*}{0.071} \\
\hline Absent & 99 (9.34) & $10(76.9)$ & $7(77.8)$ & $1(100)$ & \\
\hline
\end{tabular}

\section{TABLE 3: Association of clinicopathological parameters with histological type}

Chi-square test was applied.

${ }^{*} \mathrm{p}$-Value significant as $\leq 0.05$.

FIGO, International Federation of Gynecology and Obstetrics; T, tumor; N, nodal

\section{Discussion}

In this study, we evaluated the clinicopathological characteristics of different types of EC. We found that type $1 \mathrm{EC}$ was more frequent compared to type $2 \mathrm{EC}$. Type $2 \mathrm{EC}$ was noted to have a significantly higher 
Type $2 \mathrm{EC}$ is considered more aggressive compared to type 1 cancer; however, these are less frequent. A study from India involving 84 cases of EC revealed that $11.9 \%$ were type $2 \mathrm{ECs}$, with more than half showing myometrial invasion [6]. In our study, type $2 \mathrm{EC}$ comprised $17.8 \%$ of cases, and myometrial invasion was seen in all cases. Moreover, $56.5 \%$ cases depicted more than half of myometrial invasion, and adnexal involvement was seen in $21.7 \%$ cases. In our study, serous carcinoma was the most common type of type 2 EC followed by carcinosarcoma.

In this study, we noted that the majority of the patients were over 50 years of age and postmenopausal. Early age EC, especially in the presence of family history or synchronous/metachronous cancers, raises concerns about the microsatellite instability pathway induced EC [7].

We used the three-tiered FIGO grading system to grade type $1 \mathrm{EC}$ in our study, and $51.9 \%$ cases were grade 1. Recently, FIGO recommended a binary scheme to grade endometrioid carcinoma, according to which grade 1 and grade 2 tumors are now known as low grade, and grade 3 tumors are defined as high grade [8]. According to this new classification, $48.1 \%$ of endometrioid carcinomas in our study were high grade.

A study conducted in Egypt compared the histological features of type 1 and 2 ECs and concluded that nuclear grade, mitotic activity, and low apoptotic count are features that predict type $2 \mathrm{EC}$ histologically. In their investigation, $73.1 \%$ cases were type $1 \mathrm{ECs}$ [9]. In our study, $82.2 \%$ cases were type $1 \mathrm{ECs}$. Song et al. studied 59 cases of stage I and II ECs, among which $83 \%$ were stage I, whereas $39 \%$ were grade 1 [10]. We found that $42.6 \%$ were grade $1 \mathrm{EC}$ and $72.8 \%$ were FIGO stage I. A 10 -year comparative study including 266 cases of type $2 \mathrm{EC}$ revealed a significant association of type $2 \mathrm{EC}$ with higher tumor dimension, the depth of myometrial invasion, tumor stage, and disease recurrence [11]. We found a significant association of type 2 EC with nodal metastasis, lymphovascular invasion and adnexal involvement; however, the association with tumor stage was not significant in our study. This lack of significance may be explained by the small number of type 2 EC cases in our study.

Lymphovascular invasion is one of the important pathways of tumor spread. We found that type $2 \mathrm{EC}$ had a significantly higher rate of lymphovascular invasion compared to type $1 \mathrm{EC}$. A unique pathway of tumor spread in endometrial malignancies is transtubal spread, by which shed tumor cells from the endometrial cavity travel through the fallopian tubes to seed the pelvic cavity and involve the adnexa, even in the absence of significant myometrial invasion. In our study, adnexal involvement was noted in $21.7 \%$ of type 2 $\mathrm{EC}$, which is alarming.

One of the major limitations of the study was the small sample size, and in particular the number of cases of type $2 \mathrm{EC}$ was low. Second, the study design was retrospective and there was a lack of clinical follow-up. Large-scale prospective studies of type $2 \mathrm{EC}$ are needed to evaluate cancer-specific survival in type $2 \mathrm{EC}$ in our population. Moreover, recently a molecular-based classification of EC is proposed where four genomic subtypes of EC were identified and it was recommended that EC should be classified into the following categories: (1) p53 wild-type/copy number low, (2) p53 abnormal/copy number high, (3) polymerase E mutant, and (4) mismatch repair deficient [8]. Therefore, we recommended that prospective studies looking at the prognostic significance of this genomic classification should be conducted in our population.

\section{Conclusions}

We found a low frequency of type $2 \mathrm{EC}$ in our study, which is considered an aggressive variant of EC. We also found that type 2 ECs were associated with a higher frequency of nodal metastasis, which is one of the most important prognostic parameters of ECs. Similarly, lymphovascular invasion that is an indicator of regional and systemic metastasis was also noted more frequently in type $2 \mathrm{ECs}$ along with adnexal involvement indicating poor prognostic profile of type $2 \mathrm{ECs}$.

\section{Additional Information \\ Disclosures}

Human subjects: Consent was obtained by all participants in this study. N/A issued approval N/A. Animal subjects: All authors have confirmed that this study did not involve animal subjects or tissue. Conflicts of interest: In compliance with the ICMJE uniform disclosure form, all authors declare the following: Payment/services info: All authors have declared that no financial support was received from any organization for the submitted work. Financial relationships: All authors have declared that they have no financial relationships at present or within the previous three years with any organizations that might have an interest in the submitted work. Other relationships: All authors have declared that there are no other relationships or activities that could appear to have influenced the submitted work.

\section{References}

1. Bokhman JV: Two pathogenetic types of endometrial carcinoma . Gynecol Oncol. 1983, 15:10-17. 


\section{Cureus}

10.1016/0090-8258(83)90111-7

2. Brinton LA, Felix AS, McMeekin DS, et al.: Etiologic heterogeneity in endometrial cancer: evidence from a Gynecologic Oncology Group trial. Gynecol Oncol. 2013, 129:277-284. 10.1016/j.ygyno.2013.02.023

3. Hashmi AA, Hussain ZF, Irfan M, et al.: Epidermal growth factor receptor (EGFR) overexpression in endometrial carcinoma: association with histopathologic parameters. Surg and Exp Pathol. 2019, 2:8.

4. Hashmi AA, Hussain ZF, Qadri A, et al.: Androgen receptor expression in endometrial carcinoma and its correlation with clinicopathologic features. BMC Res Notes. 2018, 11:289. 10.1186/s13104-018-3403-9

5. Hutt S, Tailor A, Ellis P, Michael A, Butler-Manuel S, Chatterjee J: The role of biomarkers in endometrial cancer and hyperplasia: a literature review. Acta Oncol. 2019, 58:342-352. 10.1080/0284186X.2018.1540886

6. Lobo FD, Thomas E: Type II endometrial cancers: a case series . J Midlife Health. 2016, 7:69-72. 10.4103/0976-7800.185335

7. Hashmi AA, Mudassir G, Hashmi RN, et al.: Microsatellite instability in endometrial carcinoma by immunohistochemistry, association with clinical and histopathologic parameters. Asian Pac J Cancer Prev. 2019, 20:2601-2606. 10.31557/APJCP.2019.20.9.2601

8. Soslow RA, Tornos C, Park KJ, et al.: Endometrial carcinoma diagnosis: use of FIGO grading and genomic subcategories in clinical practice: recommendations of the International Society of Gynecological Pathologists. Int J Gynecol Pathol. 2019, 38:64-74. 10.1097/PGP.0000000000000518

9. Abd El-Wahed MM, Abdou AG, Al-Sharaky DR, Kasem HA: Clinicopathological differences between type I and type II endometrial carcinoma. Menoufia Med J. 2017, 30:946-951.

10. Song SH, Lee JK, Oh MJ, Hur JY, Park YK, Saw HS: Clinicopathologic Characteristics and prognostic factors of stage I and II endometrial cancer of the uterus: a study of 59 cases. Obstet Gynecol Sci. 2006, 49:12671275.

11. Feinberg J, Albright B, Black J, et al.: Ten-year comparison study of type 1 and 2 endometrial cancers: risk factors and outcomes. Gynecol Obstet Invest. 2019, 84:290-297. 10.1159/000493132 